\title{
An investigation into the correlation between pre-pregnancy body mass index and birth outcomes of newborn in Jersey
}

\author{
Urszula Sliwka $^{1,2}, \underline{\text { Katarzyna Przybylowicz }}{ }^{2}$, Neil MacLachlan ${ }^{3}$, Jakub Morze ${ }^{2}$, \\ Anna Danielewicz and Adam Surma ${ }^{2}$ \\ ${ }^{1}$ Department of Human Nutrition, University of Warmia and Mazury in Olsztyn, 10-718 Olsztyn, Poland Maternity, \\ Health and Social Services Department (HSSD), Jersey, Jersey, United Kingdom, \\ ${ }^{2}$ Department of Human Nutrition, University of Warmia and Mazury in Olsztyn, Olsztyn, Poland and \\ ${ }^{3}$ Jersey General Hospital; Health and Social Services Department (HSSD), Jersey, United Kingdom
}

\begin{abstract}
The role of nutritional status of pregnant women and birth outcomes is ambiguous. Recent studies show that pre-pregnancy body weight is equally important as weight gain during pregnancy. Body mass index (BMI) is the most accessible and easy to check a nutritional status index, which may help to control the gestation and predict infant health outcome. This study aimed to examine the associations between pre-pregnancy body mass index and the infant birth parameters. A presented observational study was offered to 200 pregnant women from Antenatal Clinic at Jersey General Hospital in 2017. Total number of 83 women agreed to take part in this project. Diet, lifestyle, socio-economic, and demographic data were obtained from participants. Delivery and birth data were taken from hospital records. Offspring feeding data and selected anthropometric measurements for mothers and their newborns were also collected. Differences between BMI for delivery type and way of feeding were verified with chi-square test. Differences and correlation between maternal BMI and newborn outcomes were verified with Kruskal-Wallis' test and Spearman's rank test. Mean BMI of mothers included to the study was $22.8 \pm 4.4$ with mean weight $61.9 \pm 11.6$. Before pregnancy BMI was normal in $67 \%$ women and about $23 \%$ was overweight or obese. We do not observed differences between delivery type and way of feeding during first 48 hours, and women in BMI categories. Also no differences and correlation were observed for the Apgar score, gestational age of birth, and newborn's weight and length at birth. However, newborn weight correlated with newborn length $(r=0.433)$ and gestational age $(\mathrm{r}=0.568)$ at birth. Concluding, the maternal pre-pregnancy BMI was not correlated with type of delivery, way of feeding and newborn outcomes. Previous studies show that high pre-pregnancy maternal BMI may be associated with adverse offspring outcomes at birth and later life. Future extended research is needed to explain these relations, with inclusion of the specific factors as maternal diet, lifestyle and ethnicity.
\end{abstract}

\section{Conflict of Interest}

There is no conflict of interest. 